Comunicação Breve

Brief Communication

Felipe Moreti ${ }^{1}$

Maria Emília Barros de Ávila²

Clara Rocha ${ }^{2}$

Maria Cristina de Menezes Borrego ${ }^{2}$

Gisele Oliveira²

Mara Behlau'

Descritores

Voz

Qualidade de vida

Estudos de avaliação

Protocolos

Música

Questionários

Keywords

Voice

Quality of life

Evaluation studies

Protocols

Music

Questionnaires

Endereço para correspondência:

Felipe Moreti

R. Machado Bittencourt, 361/1001, Vila Clementino, São Paulo (SP), Brasil, CEP: 04044-905.

E-mail: felipemoreti@uol.com.br

Recebido em: 2/9/2011

Aceito em: 12/12/2011

\section{Influência da queixa e do estilo de canto na desvantagem vocal de cantores}

\section{Influence of complaints and singing style in singers voice handicap}

\section{RESUMO}

O objetivo deste trabalho foi verificar se a diferença nos estilos de canto e a presença de queixas de voz influenciam na percepção de desvantagem vocal de cantores. Foram selecionados 118 protocolos de autoavaliação da desvantagem vocal no canto referentes a 17 cantores populares com queixas vocais, 42 populares sem queixas, 17 clássicos com queixas e 42 clássicos sem queixas. Os grupos eram semelhantes em relação à idade, gênero e naipes. Os protocolos utilizados, Índice de Desvantagem para o Canto Moderno e Índice de Desvantagem para o Canto Clássico, apresentam questões específicas para os respectivos estilos de canto e são compostos por 30 itens divididos igualmente em três subescalas: incapacidade (domínio funcional), desvantagem (domínio emocional) e defeito (domínio orgânico), respondidos de acordo com a frequência de ocorrência. Cada subescala apresenta valor máximo de 40 pontos, e o total corresponde a 120 pontos. Quanto maior a pontuação, maior a desvantagem vocal percebida. Para análise estatística, utilizou-se o teste ANOVA, com significância de 5\%. Cantores clássicos e populares referiram maior defeito, seguido por incapacidade e desvantagem. Contudo, o grau dessa percepção nesse grupo variou de acordo com o estilo de canto e a presença de queixas vocais. Cantores clássicos com queixas vocais apresentaram maior desvantagem vocal que os cantores populares também com queixas. Clássicos sem queixas relataram menor desvantagem que populares também sem queixas. Isso evidencia que o cantor clássico tem maior percepção sobre sua própria voz e que uma alteração vocal nesse grupo pode causar maior desvantagem vocal do que no grupo de cantores populares.

\begin{abstract}
The aim of this research was to verify whether the difference of singing styles and the presence of vocal complaints influence the perception of voice handicap of singers. One hundred eighteen singing voice handicap self-assessment protocols were selected: 17 popular singers with vocal complaints, 42 popular singers without complaints, 17 classic singers with complaints, and 42 classic singers without complaints. The groups were similar regarding age, gender and voice types. Both protocols used - Modern Singing Handicap Index (MSHI) and Classical Singing Handicap Index (CSHI) - have specific questions to their respective singing styles, and consist of 30 items equally divided into three subscales: disability (functional domain), handicap (emotional domain) and impairment (organic domain), answered according to the frequency of occurrence. Each subscale has a maximum of 40 points, and the total score is 120 points. The higher the score, the higher the singing voice handicap perceived. For statistical analysis, we used the ANOVA test, with 5\% of significance. Classical and popular singers referred higher impairment, followed by disability and handicap. However, the degree of this perception varied according to the singing style and the presence of vocal complaints. The classical singers with vocal complaints showed higher voice handicap than popular singers with vocal complaints, while the classic singers without complaints reported lower handicap than popular singers without complaints. This evidences that classical singers have higher perception of their own voice, and that vocal disturbances in this group may cause greater voice handicap when compared to popular singers.
\end{abstract}

Trabalho realizado no Centro de Estudos da Voz - CEV - São Paulo (SP), Brasil.

(1) Centro de Estudos da Voz - CEV - São Paulo (SP), Brasil; Departamento de Fonoaudiologia, Universidade Federal de São Paulo - UNIFESP - São Paulo (SP), Brasil.

(2) Centro de Estudos da Voz - CEV - São Paulo (SP), Brasil

Conflito de interesses: Não 


\section{INTRODUÇÃO}

Na área de voz, o Voice Handicap Index - VHI é um dos mais conhecidos e utilizados protocolos de autoavaliação do impacto de um problema de $\mathrm{voz}^{(1)}$, desenvolvido nos Estados Unidos $^{(2)} \mathrm{e}$ validado em quase 20 países $^{(3)}$, inclusive no Brasil ${ }^{(4)}$, como Índice de Desvantagem Vocal - IDV. Apesar da indiscutível validade e confiabilidade do VHI, sua sensibilidade para avaliar cantores é pobre, uma vez que os fatores associados à percepção do indivíduo sobre sua desvantagem na voz cantada não são abordados neste protocolo ${ }^{(5)}$.

Para atender a essa população, foram desenvolvidas adaptações do VHI para a voz cantada, em inglês ${ }^{(6)} \mathrm{e}$ francês ${ }^{(7)}$. Cantores populares e clássicos possuem diferentes formações musicais, diferindo em suas técnicas, exigências vocais e percepção de suas próprias vozes, e por isso merecem análises de formas diferentes, para uma maior especificidade da avaliação. Recentemente, o foniatra italiano Franco Fussi propôs duas versões específicas para o estilo de canto clássico e popular: Modern Singing Handicap Index - MSHI, para cantores populares e Classical Singing Handicap Index - CSHI, para cantores clássicos ${ }^{(8)}$, traduzidas e culturalmente adaptadas para o português brasileiro como Índice de Desvantagem para o Canto Moderno - $\operatorname{IDCM}^{(9)}$ (Anexo 1) e Índice de Desvantagem para o Canto Clássico - IDCC ${ }^{(10)}$ (Anexo 2 ), respectivamente. Ainda não se sabe se as desvantagens de ambos os estilos são comparáveis.

Desta forma, o objetivo desta pesquisa foi verificar se a diferença dos estilos de canto e a presença de queixas de voz influenciam na percepção de desvantagem vocal de cantores.

\section{MÉTODOS}

A pesquisa foi aprovada pelo comitê de ética institucional, sob o número 0819/11 e utilizou um banco dados de protocolos com a devida autorização do responsável pela instituição detentora destes documentos. Foram consultadas as respostas dos questionários de autoavaliação da desvantagem vocal no canto, específicos para cantores populares (IDCM) e clássicos (IDCC). Selecionou-se 118 protocolos, distribuídos em quatro grupos: 17 cantores populares com queixas vocais $-\mathrm{CpCQ}$, 42 populares sem queixas - CpSQ, 17 clássicos com queixas - CcCQ e 42 clássicos sem queixas - CcSQ. Os grupos eram demograficamente semelhantes, com média de idade de 29,5 anos ( $\mathrm{p}=1,000)$, sendo 66 mulheres - 44 sopranos e 22 contraltos - e 52 homens -26 tenores e 26 baixos - (distribuição por gênero: $\mathrm{p}=0,776$ e naipes: $\mathrm{p}=0,665$ ).

Ambos os protocolos possuem questões específicas para os respectivos estilos de canto e são compostos por 30 itens, divididos igualmente em três subescalas: incapacidade (domínio funcional), desvantagem (domínio emocional) e defeito (domínio orgânico). As questões foram respondidas de acordo com a frequência de ocorrência (nunca, quase nunca, às vezes, quase sempre e sempre). Cada subescala apresenta valor máximo de 40 pontos e o escore total do protocolo corresponde a 120 pontos. Quanto maior a pontuação, maior a desvantagem vocal percebida.

Para a análise estatística, adotou-se o nível de significância de 5\% (0,05). Foi utilizado o teste paramétrico ANOVA.

\section{RESULTADOS}

Apesar de os escores terem sido diferentes para os estilos, a ordenação da maior desvantagem para a menor foi a mesma: defeito (domínio orgânico), seguida por incapacidade (domínio funcional) e desvantagem (domínio emocional). Maiores escores foram apresentados pelo grupo CcCQ (Tabela 1).

Indivíduos com queixas vocais, de ambos os estilos, apresentaram maior desvantagem em relação aos sem queixas, em todas as subescalas (Tabela 2).

Tabela 1. Médias dos escores dos grupos nas subescalas e total do IDCM e IDCC

\begin{tabular}{|c|c|c|c|c|c|c|c|c|c|}
\hline Subescalas por grupo & & Média & Mediana & $\mathrm{DP}$ & Min & Max & $\mathrm{n}$ & IC & Valor de $p$ \\
\hline \multirow{4}{*}{ Incapacidade } & $\mathrm{CcCQ}$ & 11,7 & 11 & 6,4 & 3 & 31 & 17 & 3,1 & \multirow{4}{*}{$<0,001^{*}$} \\
\hline & $\operatorname{CcSQ}$ & 2,7 & 2 & 2,7 & 0 & 12 & 42 & 0,8 & \\
\hline & $\mathrm{CpCQ}$ & 6,0 & 6 & 3,1 & 0 & 11 & 17 & 1,5 & \\
\hline & CpSQ & 2,8 & 3 & 2,2 & 0 & 8 & 42 & 0,7 & \\
\hline \multirow{2}{*}{ Desvantagem } & $\mathrm{CcCQ}$ & 7,6 & 7 & 6,3 & 0 & 26 & 17 & 3,0 & \multirow{2}{*}{$<0,001^{*}$} \\
\hline & CcSQ & 1,6 & 1 & 2,5 & 0 & 13 & 42 & 0,8 & \\
\hline \multirow{4}{*}{ Defeito } & $\mathrm{CcCQ}$ & 11,9 & 11 & 6,1 & 2 & 28 & 17 & 2,9 & \multirow{4}{*}{$<0,001^{*}$} \\
\hline & $\operatorname{CcSQ}$ & 4,1 & 4 & 3,9 & 0 & 13 & 42 & 1,2 & \\
\hline & $\mathrm{CpCQ}$ & 10,5 & 11 & 3,4 & 6 & 17 & 17 & 1,6 & \\
\hline & CpSQ & 6,2 & 6 & 4,0 & 0 & 14 & 42 & 1,2 & \\
\hline Total & $\mathrm{CcCQ}$ & 31,2 & 29 & 17,5 & 6 & 85 & 17 & 8,3 & $<0,001^{*}$ \\
\hline
\end{tabular}

* Valores significativos $(\mathrm{p} \leq 0,05)$ - ANOVA

Legenda: $\mathrm{CcCQ}=$ grupo cantores clássicos com queixas vocais; $\mathrm{CcSQ}=$ grupo cantores clássicos sem queixas vocais; $\mathrm{CpCQ}=$ grupo cantores populares com queixas vocais; $\mathrm{CpSQ}=$ grupo cantores populares sem queixas vocais; $\mathrm{DP}=$ desvio-padrão; Min = mínimo valor do escore nos protocolos; Max = máximo valor do escore nos protocolos; IC = intervalo de confiança para a média 
Tabela 2. Médias dos escores dos indivíduos com e sem queixas vocais nas subescalas do IDCM e IDCC

\begin{tabular}{llccccccccc}
\hline Cantores & & Média & Mediana & DP & Min & Max & $\mathrm{n}$ & IC & Valor de $\mathrm{p}$ \\
\hline \multirow{2}{*}{ Com queixas vocais: } & Incapacidade & 8,9 & 9 & 5,7 & 0 & 31 & 34 & 1,9 & \\
CcCQ + CpCQ & Desvantagem & 5,2 & 4 & 5,3 & 0 & 26 & 34 & 1,8 & $<0,001^{*}$ \\
& Defeito & 11,2 & 11 & 4,9 & 2 & 28 & 34 & 1,7 & 0,5 \\
\hline \multirow{2}{*}{ Sem queixas vocais: } & Incapacidade & 2,8 & 2 & 2,5 & 0 & 12 & 84 & 0,5 \\
CcSQ + CpSQ & Desvantagem & 1,6 & 1 & 2,1 & 0 & 13 & 84 & 0,5 & $<0,001^{*}$ \\
& Defeito & 5,2 & 4 & 4,1 & 0 & 14 & 84 & 0,9 & \\
\hline
\end{tabular}

* Valores significativos $(p \leq 0,05)$ - ANOVA

Legenda: $\mathrm{CcCQ}=$ grupo cantores clássicos com queixas vocais; $\mathrm{CcSQ}=$ grupo cantores clássicos sem queixas vocais; $\mathrm{CpCQ}=$ grupo cantores populares com queixas vocais; $\mathrm{CpSQ}$ = grupo cantores populares sem queixas vocais; $\mathrm{DP}$ = desvio-padrão; Min = mínimo valor do escore nos protocolos; Max = máximo valor do escore nos protocolos; IC = intervalo de confiança para a média

\section{DISCUSSÃO}

Fatores como demanda vocal elevada, uso da voz em extremos vocais, falta de domínio técnico e limitada experiência no canto $^{(5-8)}$ podem explicar o maior desvio da subescala defeito ${ }^{(11)}$ e, consequentemente, colocar os indivíduos em uma situação de risco vocal potencial ${ }^{(6)}$. Normalmente os cantores clássicos possuem uma maior exigência e melhor técnica em relação ao uso da voz que os cantores populares ${ }^{(12)}$, o que pode gerar uma percepção mais aguçada de um problema vocal. Desta forma, cantores clássicos sem queixas referem pequena desvantagem vocal, enquanto os cantores clássicos com queixas referem maior desvantagem, pois possivelmente um problema de voz poder colocar sua carreira em elevado risco ${ }^{(10)}$. Já no canto popular, pequenas alterações vocais podem inclusive ser usadas para composição do estilo e assinatura vocal do cantor ${ }^{(9)}$.

A presença da queixa de voz foi determinante na manifestação da desvantagem vocal ${ }^{(13)}$. Isso mostra a sensibilidade de cada protocolo de acordo com o estilo para as populações de cantores populares e clássicos com queixas vocais ${ }^{(11,14)}$.

\section{CONCLUSÃO}

Cantores clássicos e populares referem maior defeito (domínio orgânico), seguido por incapacidade (domínio funcional) e desvantagem (domínio emocional). Contudo, o grau dessa percepção varia de acordo com o estilo de canto e a presença de queixas vocais.

Os cantores clássicos com queixas vocais apresentam maior desvantagem vocal que os cantores populares também com queixas vocais, enquanto os clássicos sem queixas relatam menor desvantagem que os populares também sem queixas. Isso evidencia que o cantor clássico tem uma maior percepção sobre sua própria voz e que uma alteração vocal nesse grupo pode causar maior desvantagem vocal do que no grupo de cantores populares.

\section{REFERÊNCIAS}

1. Gräßel E, Hoppe U, Rosanowski F. Graduierung des Voice-HandicapIndex. HNO. 2008;56(12):1221-8.

2. Jacobson BH, Johnson A, Grywalski C, Silbergleit A, Jacobson G, Benninger MS, et al. The Voice Handicap Index (VHI): development and validation. Am J Speech Lang Pathol. 1997;6(3):66-70.

3. Verdonck-de Leeuw IM, Kuik DJ, De Bodt M, Guimaraes I, Holmberg $\mathrm{EB}$, Nawka T, et al. Validation of the voice handicap index by assessing equivalence of European translations. Folia Phoniatr Logop. 2008;60(4):173-8.

4. Behlau M, Alves dos Santos LM, Oliveira G. Cross-cultural adaptation and validation of the voice handicap index into brazilian portuguese. $\mathrm{J}$ Voice. 2011;25(3):354-9.

5. Cohen SM, Noordzij JP, Garrett CG, Ossoff RH. Factors associated with perception of singing voice handicap. Otolaryngol Head Neck Surg. 2008;138(4):430-4.

6. Cohen SM, Jacobson BH, Garrett CG, Noordzij JP, Stewart MG, Attia A, et al. Creation and validation of the Singing Voice Handicap Index. Ann Oto Rhino Laryngol. 2007;116(6):402-6.

7. Morsomme D, Gaspar M, Jamart J, Remacle M, Verduyckt I. Adaptation du Voice Handicap Index à la voix chantée. Rev Laryngol Otol Rhinol (Bord). 2007;128(5):305-14.

8. Fussi F, Fuschini T. Foniatria artistica: la presa in carico foniatricologopedica del cantante classico e moderno. Audiologia \& Foniatria. 2008;13(1-2):4-28

9. Moreti F, Rocha C, Borrego MC, Behlau M. Desvantagem vocal no canto: análise do protocolo Índice de Desvantagem para o Canto Moderno - IDCM. Rev Soc Bras Fonoaudiol. 2011;16(2):146-51.

10. Ávila ME, Oliveira G, Behlau M. Índice de desvantagem vocal no canto clássico (IDCC) em cantores eruditos. Pró-Fono. 2010;22(3):221-6.

11. Jotz GP, Bramati O, Schimidt VB, Dornelles S, Gigante LP. Aplicação do "Voice Handicap Index" em Coralistas. Arq Otorrinolaringol. 2002;6(4):260-4.

12. Zampieri SA, Behlau M, Brasil OO. Análise de cantores de baile em estilo de canto popular e lírico: perceptivo-auditiva, acústica e da configuração laríngea. Rev Bras Otorrinolaringol. 2002;68(3):378-86.

13. Murry T, Zschommler A, Prokop J. Voice Handicap in Singers. J Voice. 2009;23(3):376-9.

14. Rosen CA, Murry T. Voice handicap index in singers. J Voice. 2000;14(3):370-7. 
Anexo 1. Versão brasileira do protocolo Modern Singing Handicap Index - MSHI(8), intitulada Índice de Desvantagem para o Canto Moderno - IDCM ${ }^{(9)}$

\section{Índice de Desvantagem para o Canto Moderno - IDCM}

Marque a resposta que indica o quanto você compartilha da mesma experiência:

$0=$ nunca 1 = quase nunca 2 = às vezes 3 = quase sempre $4=$ sempre

Incapacidade - o impacto do problema de voz nas atividades profissionais

\begin{tabular}{|c|c|c|c|c|c|c|}
\hline 1 & Sinto minha voz cansada desde o começo de uma apresentação & 0 & 1 & 2 & 3 & 4 \\
\hline 2 & Minha voz fica cansada ou alterada durante a apresentação & 0 & 1 & 2 & 3 & 4 \\
\hline 3 & Tenho que ajustar a minha técnica vocal, porque o problema de voz prejudica a minha emissão & 0 & 1 & 2 & 3 & 4 \\
\hline 4 & Meu problema vocal me obriga a modificar as músicas, limitar meu repertório ou mesmo mudar o tom & 0 & 1 & 2 & 3 & 4 \\
\hline 5 & Por causa do meu problema de voz sou forçado a limitar meu tempo de estudo/ensaio & 0 & 1 & 2 & 3 & 4 \\
\hline 6 & Sinto dificuldade nas apresentações por causa das alterações no meu rendimento vocal & 0 & 1 & 2 & 3 & 4 \\
\hline 7 & Não consigo fazer duas ou mais apresentações consecutivas & 0 & 1 & 2 & 3 & 4 \\
\hline 8 & Preciso da ajuda do operador de som para mascarar meu problema de voz & 0 & 1 & 2 & 3 & 4 \\
\hline 9 & Preciso tomar remédios continuamente para mascarar meu problema de voz & 0 & 1 & 2 & 3 & 4 \\
\hline 10 & Meu problema vocal me obriga a limitar o uso social da voz & 0 & 1 & 2 & 3 & 4 \\
\hline
\end{tabular}

Desvantagem - o impacto psicológico do problema de voz

\begin{tabular}{|c|c|c|c|c|c|c|}
\hline 1 & Minha ansiedade antes das apresentações está maior que a habitual & 0 & 1 & 2 & 3 & 4 \\
\hline 2 & As pessoas com as quais convivo não compreendem minha queixa de voz & 0 & 1 & 2 & 3 & 4 \\
\hline 3 & As pessoas com as quais convivo têm criticado a minha voz & 0 & 1 & 2 & 3 & 4 \\
\hline 4 & Meu problema de voz me deixa nervoso e/ou menos sociável & 0 & 1 & 2 & 3 & 4 \\
\hline 5 & Fico preocupado quando me pedem para repetir um vocalize ou uma frase musical & 0 & 1 & 2 & 3 & 4 \\
\hline 6 & Sinto que minha carreira está em risco por causa do meu problema de voz & 0 & 1 & 2 & 3 & 4 \\
\hline 7 & Colegas, empresários e críticos já perceberam minhas dificuldades vocais & 0 & 1 & 2 & 3 & 4 \\
\hline 8 & Sou obrigado a cancelar alguns compromissos profissionais por causa da voz & 0 & 1 & 2 & 3 & 4 \\
\hline 9 & Evito agendar futuros compromissos profissionais & 0 & 1 & 2 & 3 & 4 \\
\hline 10 & Evito conversar com as pessoas & 0 & 1 & 2 & 3 & 4 \\
\hline
\end{tabular}

Defeito - autopercepção das características de minha voz

\begin{tabular}{|c|c|c|c|c|c|c|}
\hline 1 & Tenho problemas com o controle da respiração para o canto & 0 & 1 & 2 & 3 & 4 \\
\hline 2 & Meu rendimento vocal varia durante o dia & 0 & 1 & 2 & 3 & 4 \\
\hline 3 & Sinto que minha voz está fraca ou tem ar na voz & 0 & 1 & 2 & 3 & 4 \\
\hline 4 & Sinto minha voz rouca & 0 & 1 & 2 & 3 & 4 \\
\hline 5 & Sinto que tenho que forçar minha voz para produzir os sons & 0 & 1 & 2 & 3 & 4 \\
\hline 6 & Meu rendimento vocal varia de modo imprevisível durante as apresentações & 0 & 1 & 2 & 3 & 4 \\
\hline 7 & Tento modificar minha voz para melhorar a qualidade & 0 & 1 & 2 & 3 & 4 \\
\hline 8 & Cantar está sendo uma tarefa difícil ou cansativa & 0 & 1 & 2 & 3 & 4 \\
\hline 9 & Minha voz fica pior à noite & 0 & 1 & 2 & 3 & 4 \\
\hline 10 & Minha voz fica facilmente cansada durante as apresentações & 0 & 1 & 2 & 3 & 4 \\
\hline
\end{tabular}

Nota: para o cálculo dos escores parciais ou total, deve-se realizar a somatória simples nos domínios ou de todo o protocolo. Para passar para base 100, dividir o escore total ou parcial do domínio por 120 (que é o escore máximo deste protocolo). 
Anexo 2. Versão brasileira do protocolo Classical Singing Handicap Index - CSHI ${ }^{(8)}$, intitulada Índice de Desvantagem para o Canto Clássico - IDCC ${ }^{(10)}$

\section{Índice de Desvantagem para o Canto Clássico - IDCC}

Marque a resposta que indica o quanto você compartilha da mesma experiência:

$0=$ nunca $1=$ quase nunca 2 = às vezes $3=$ quase sempre $4=$ sempre

Incapacidade - o impacto do problema de voz nas atividades profissionais

\begin{tabular}{|c|c|c|c|c|c|c|}
\hline 1 & Tenho dificuldades durante as apresentações por causa de alterações de meu rendimento vocal & 0 & 1 & 2 & 3 & 4 \\
\hline 2 & Tenho levado mais tempo para aquecer a minha voz & 0 & 1 & 2 & 3 & 4 \\
\hline 3 & Minha voz fica cansada ou alterada durante as apresentações & 0 & 1 & 2 & 3 & 4 \\
\hline 4 & Tenho que mudar aspectos da minha técnica vocal, porque o problema de voz prejudica a minha emissão & 0 & 1 & 2 & 3 & 4 \\
\hline 5 & Meu problema vocal me obriga a modificar músicas ou limitar meu repertório & 0 & 1 & 2 & 3 & 4 \\
\hline 6 & Por causa do meu problema de voz sou forçado a limitar meu tempo habitual de estudo/ensaio & 0 & 1 & 2 & 3 & 4 \\
\hline 7 & Sou obrigado a fazer períodos de descanso vocal mais longos entre as apresentações ou produções & 0 & 1 & 2 & 3 & 4 \\
\hline 8 & Preciso evitar dinâmicas de volume em "pianíssimo" & 0 & 1 & 2 & 3 & 4 \\
\hline 9 & Preciso tomar remédios continuamente para mascarar meu problema de voz & 0 & 1 & 2 & 3 & 4 \\
\hline 10 & Meu problema vocal me obriga a limitar o uso social da voz & 0 & 1 & 2 & 3 & 4 \\
\hline
\end{tabular}

Desvantagem - o impacto psicológico do problema de voz

\begin{tabular}{|c|c|c|c|c|c|c|}
\hline 1 & Minha ansiedade antes das apresentações está maior que a habitual & 0 & 1 & 2 & 3 & 4 \\
\hline 2 & As pessoas com as quais convivo não compreendem minha queixa de voz & 0 & 1 & 2 & 3 & 4 \\
\hline 3 & As pessoas com as quais convivo têm criticado a minha voz & 0 & 1 & 2 & 3 & 4 \\
\hline 4 & Meu problema de voz me deixa nervoso ou menos sociável & 0 & 1 & 2 & 3 & 4 \\
\hline 5 & Fico preocupado quando me pedem para repetir um vocalise ou uma frase musical & 0 & 1 & 2 & 3 & 4 \\
\hline 6 & Sinto que minha carreira está em risco por causa do meu problema de voz & 0 & 1 & 2 & 3 & 4 \\
\hline 7 & Colegas, diretores e críticos já notaram minhas dificuldades vocais & 0 & 1 & 2 & 3 & 4 \\
\hline 8 & Sou obrigado a cancelar alguns compromissos profissionais por causa da voz & 0 & 1 & 2 & 3 & 4 \\
\hline 9 & Evito agendar futuros compromissos profissionais & 0 & 1 & 2 & 3 & 4 \\
\hline 10 & Evito conversar com as pessoas & 0 & 1 & 2 & 3 & 4 \\
\hline
\end{tabular}

Defeito - autopercepção das características de minha voz

\begin{tabular}{|c|c|c|c|c|c|c|}
\hline 1 & Tenho dificuldades com o controle respiratório por causa do meu problema de voz & 0 & 1 & 2 & 3 & 4 \\
\hline 2 & Meu rendimento vocal varia durante o dia & 0 & 1 & 2 & 3 & 4 \\
\hline 3 & Acho que minha voz cantada está rouca ou ruidosa & 0 & 1 & 2 & 3 & 4 \\
\hline 4 & Tenho dificuldades em sustentar as notas (quebra de nota) & 0 & 1 & 2 & 3 & 4 \\
\hline 5 & Minha extensão vocal reduziu ou mudou & 0 & 1 & 2 & 3 & 4 \\
\hline 6 & Tenho dificuldades para equilibrar a ressonância ou os registros vocais & 0 & 1 & 2 & 3 & 4 \\
\hline 7 & Cantar tem sido difícil ou cansativo e tenho que forçar a voz para produzir os sons & 0 & 1 & 2 & 3 & 4 \\
\hline 8 & Minha qualidade vocal piora durante as apresentações & 0 & 1 & 2 & 3 & 4 \\
\hline 9 & Após as apresentações, minha voz fica cansada ou alterada & 0 & 1 & 2 & 3 & 4 \\
\hline 10 & Meu rendimento vocal piora em alguns momentos do dia & 0 & 1 & 2 & 3 & 4 \\
\hline
\end{tabular}

Nota: para o cálculo dos escores parciais ou total, deve-se realizar a somatória simples nos domínios ou de todo o protocolo. Para passar para base 100, dividir o escore total ou parcial do domínio por 120 (que é o escore máximo deste protocolo). 